\title{
Sensitized Photooxygenation of Cholesterol and Pseudo- cholesterol Derivatives via Singlet Oxygen
}

\author{
Wu Shuping ${ }^{1}$, Jiang Zhiqin ${ }^{2, *}$, Li Heting ${ }^{1}$, Yang $\mathrm{Li}^{2}$ and Zeng Daixun ${ }^{2}$ \\ ${ }^{1}$ Research Laboratory of Photochemistry, Tongji University, Shanghai 200092, P. R. China. \\ Tel.: +86 21 65982593, Fax: +86 21 65980591, E-mail: jzqcdtju@ online.sh.cn \\ ${ }^{2}$ National Laboratory of Applied Organic Chemistry at Lanzhou University, Lanzhou 730000, P. R. \\ China.
}

*Author to whom correspondence should be addressed. E-mail: jzqcdtju@online.sh.cn

Received: 30 September 1998; in revised form 4 September 2000 / Accepted: 19 September 2000 / Published: 16 January 2001

\begin{abstract}
Substituted cholesterols and 7-substituted pseudocholesterols undergo a facile photooxygenation sensitized by 9, 10-dicyanoanthracene (DCA) and lumiflavin (LF) to give similar, oppositely-positioned enol derivatives. Both steroids showed the same reaction pattern associated with the endocyclic 5- and 4-olefin units, respectively. The reaction was proposed to proceed via the ene reaction of singlet oxygen and subsequent rearrangement of the initially formed $5 \alpha$-hydroperoxides.
\end{abstract}

Keywords: Cholesterol and pseudocholesterol derivatives, ene reaction of singlet oxygen, photochemical damage.

\section{Introduction}

Photochemical damage of biological molecules by reactive oxygen species $\left({ }^{1} \mathrm{O}_{2}, \mathrm{O}_{2}{ }^{-}\right.$, $\mathrm{HO} \cdot$, RO. , $[\mathrm{O}]$ etc.) have received increasing attention recently [1,2]. Studies indicate that a number of biological pigments e.g. hematoporphyrin, riboflavin and chlorophyll etc. can readily sensitize the photooxygenation of $\Delta^{5}$-steroids such as cholesterol, which may cause some diseases or damage to biological organs. One of the effects found was an increase the brittleness of the cell membrane, which leads to hemolysis [3]. It was also verified that oxygen can affect the synthesis and metabolism of 
pregnenolone [4]. In most cases, the sensitized photooxygenations of cholesterol and other steroids involve singlet oxygen reactions [3-5]. However, the structure-reactivity features of steroids in these photooxygenations and their photochemical damage are still less well understood. In this work, 9,10dicyanoanthracene (DCA) and lumiflavin (LF) are selected as new sensitizers to further study the photooxygenation products and their distributions in cholesterol derivatives 1-3 and pseudo-cholesterol analogs 4-5, and potential influence of photochemical lesions to $\Delta$-steroids.<smiles>[R9]C1CC[C@]2(C)C(=CC[C]3C(C)C(C)([C@H](C)S)CCC32)C1</smiles>

$1 \mathrm{R}=\mathrm{H}$

$2 \mathrm{R}=\mathrm{CH}_{3}$

$3 \mathrm{R}=\mathrm{CH}_{3} \mathrm{CO}$

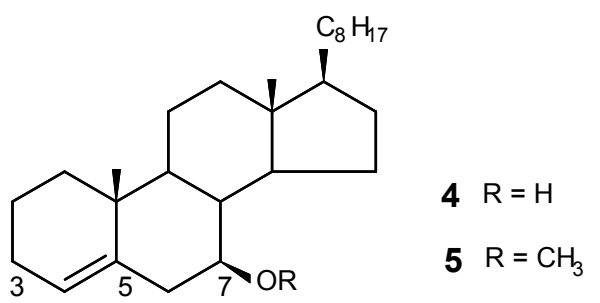<smiles>N#Cc1c2ccccc2c(C#N)c2ccccc12</smiles>

DCA<smiles>Cc1cc2nc3c(=O)[nH]c(=O)nc-3n(CO)c2cc1C</smiles>

$\mathbf{R F}$<smiles>Cc1cc2nc3c(=O)[nH]c(=O)nc-3n(C)c2cc1C</smiles>

LF

\section{Results and Discussion}

Riboflavin (RF) is known as a typical ${ }^{1} \mathrm{O}_{2}$ sensitizer, but its light stability is not very good because of facile cleavage of the polyhydroxy-containing side chain. We found that the light stability of LF is better than its RF derivatives, hence using LF may be beneficial in the title reaction. DCA not only has high light stability, but also is frequently used as an electron transfer sensitizer for aromatic compounds [6]. However, Foote [7] and the authors [8] demonstrated that DCA also is a good sensitizer to initiate the ${ }^{1} \mathrm{O}_{2}$ reaction of alicyclic olefins and $\Delta$-steroids. It was found in this work that in the presence of DCA or LF, 1-3 can undergo a facile photooxygenation in solution such as acetonitrile and pyridine to give the 6-en-5á - and 5-en-7( $\alpha, \beta)$-hydroperoxides, 1'a-3'a and 1'b-3'b, respectively, with good conversions (80-90\%). Since these hydroperoxides are not very stable at room temperature, they were treated with $\mathrm{Ph}_{3} \mathrm{P}$ and reduced to the corresponding enols $\mathbf{1 a - 3 a}$ and $\mathbf{1 b}-\mathbf{3 b}$. The reaction is represented as follows: 


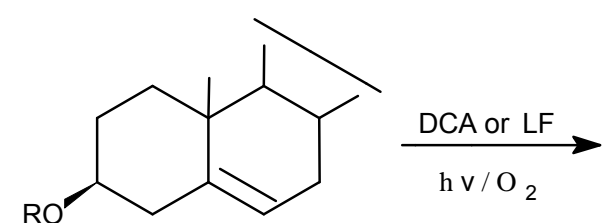

$1-3$<smiles>[R9]C1CCC2(C)C(C)C(C)C=C[C@]2(Oc2ccccc2)C(C)C1</smiles><smiles>[R9]C1CCC2(C)C(C)C(C)C=C[C@]2(O)C1</smiles>

$1 a-3 a$<smiles>[R9]C1CCC2(C)C(=C[C@H](O)C(C)C2C)C1</smiles>

$1 b-3 b$

The data for the (a, b) types of products and their distribution for the DCA- and LF-sensitized photooxygenations of $\mathbf{1}$ - $\mathbf{5}$ are listed in Table 1. It can be seen from this data that the reactions of the substituted cholesterols 1-3 give similar products, i.e enol derivatives of types $\mathbf{a}$ - and $\mathbf{b}$ - and 1a-3a are formed stereospecifically, whereas $\mathbf{1 b}-\mathbf{3 b}$ is a mixture of $7 \alpha$ - and $7 \beta$-hydroxy epimers with no stereospecificity. By comparison of the conversions of 1-3 in Table 1, it seems that DCA is more reactive than LF for sensitizinge the reaction.

The title reactions lead to the major a-type of enol product with an adjacent double-bond migration, which is in accord with the ene reaction of ${ }^{1} \mathrm{O}_{2}$ and the reported sensitized photooxygenations of other Ä-steroids [4]. Accordingly, the title reaction may follow the ${ }^{1} \mathrm{O}_{2}$ pathway (see Scheme 2). Because of axial hindrance of the 19-angular methyl group at $\mathrm{C}-10$, attack at the $5 \alpha$ - orientation of the $5-\mathrm{C}=\mathrm{C}$ bond by ${ }^{1} \mathrm{O}_{2}$, which is generated from energy transfer, is more favored, producing the peroxide intermediate. A suprafacial $7 \alpha-\mathrm{H}$ shift and adjacent $\mathrm{C}=\mathrm{C}$ migration lead to $5 \alpha-\mathrm{OOH}$ product $\left(\mathbf{a}^{\prime}\right)$. In addition, the rearrangement of $\mathbf{a}^{\prime} \quad \mathbf{b}^{\prime}$ - hydroperoxide in the sensitized photooxygenations of $\Delta$-steroids is well established to proceed through allylperoxy radicals or [2,3] sigmatropic rearrangement $[9,10]$. The results in Table 1 also show that the title reaction of $\mathbf{1}$ - $\mathbf{3}$ always accompanies formation of the $\mathbf{b}$-type of enol product. The yields of $\mathbf{1 b}-\mathbf{3 b}$ varied in the range of $12-80 \%$, depending on the conditions employed. This indicates existence of the subsequent rearrangement of $\mathbf{a}^{\prime} \quad \mathbf{b}^{\prime}$ - hydroperoxide.

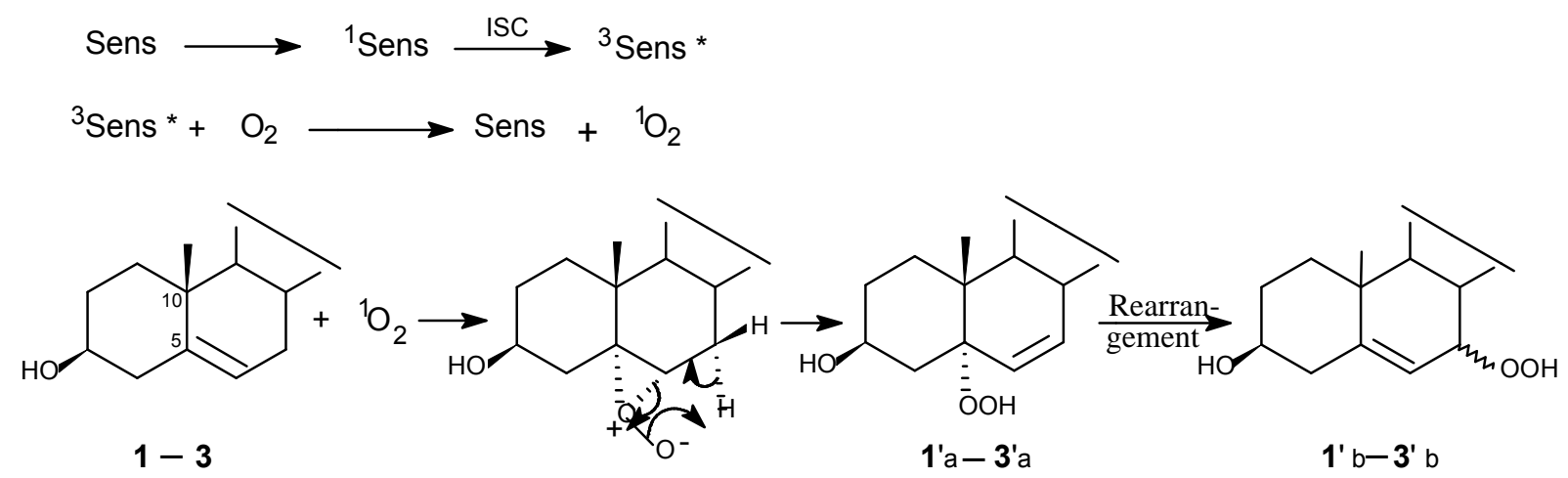

Scheme 2. 
Table 1. Sensitized photooxygenation of $\mathbf{1}$ - $\mathbf{5}$ and their product distributions.

\begin{tabular}{|c|c|c|c|c|c|c|}
\hline Steroids & Sensitizer & Solvent & $\begin{array}{c}\text { Reaction } \\
\text { Time h }\end{array}$ & $\begin{array}{c}\text { Conversion } \\
\%\end{array}$ & \multicolumn{2}{|c|}{$\begin{array}{c}\text { Relative } \\
\text { yields }(\%)\end{array}$} \\
\hline 1 & LF & $\mathrm{Py}$ & 6 & 90 & 86 & 12 \\
\hline 1 & LF & $\mathrm{MeCN}$ & 4 & 80 & 20 & 80 \\
\hline 1 & $\mathrm{DCA}^{*}$ & $\mathrm{MeCN}$ & 0.2 & 2 & 90 & 10 \\
\hline 1 & DCA & $\mathrm{MeCN}$ & 6 & 90 & 43 & 57 \\
\hline 1 & $\mathrm{RB}^{*}$ & $\mathrm{MeCN}$ & 0.2 & 2 & 90 & 10 \\
\hline 2 & LF & $\mathrm{MeCN}$ & 10 & 80 & 44 & 56 \\
\hline 2 & DCA & $\mathrm{MeCN}$ & 8 & 85 & 42 & 58 \\
\hline 3 & $\mathrm{LF}$ & $\mathrm{MeCN}$ & 12 & 74 & 72 & 28 \\
\hline 3 & DCA & $\mathrm{MeCN}$ & 7 & 84 & 68 & 32 \\
\hline 4 & DCA & $\mathrm{MeCN}$ & 5 & 47 & 80 & 20 \\
\hline 5 & DCA & $\mathrm{MeCN}$ & 7 & 50 & 76 & 24 \\
\hline
\end{tabular}

"cf. Ref. 7.

Pseudocholesterol is not found in living systems and, unlike cholesterol, it has no biological activity, although it possesses the same tetracyclic diterpene structural framework and functional groups $\left(\mathrm{C}=\mathrm{C}, \mathrm{OH}, \mathrm{CH}_{3}\right.$ and $\left.\mathrm{C}_{8} \mathrm{H}_{17}\right)$ as found in cholesterol. Interestingly, its 4-en-7 $\beta$-ol positions are oppositely arranged as compared with 5-en-3 $\beta$-ol positions in the $\mathrm{A}$ and $\mathrm{B}$ rings of cholesterol. This structural similarity may lead to a similar reaction behavior and products possessing reversed enol positions. Indeed, the results indicate that the DCA-sensitized photooxygenation of pseudocholesterols 4, 5 in MeCN under the same conditions afforded similar $\mathbf{a}-$ and $\mathbf{b}$-type hydroperoxides, 4'a-5'a and 4'b-5' $\mathbf{b}$, and subsequent 4-en-7 $\beta$-ol products, $\mathbf{4 a - 5 a}$ and $\mathbf{4 b - 5}$, after treatment with $\mathrm{Ph}_{3} \mathrm{P}$ (see Table 1). It is shown in Scheme 3 that the enol positions in the A and B rings of $\mathbf{4}$ and $\mathbf{5}$ are reversed compared to those of substituted cholesterols 1-3.

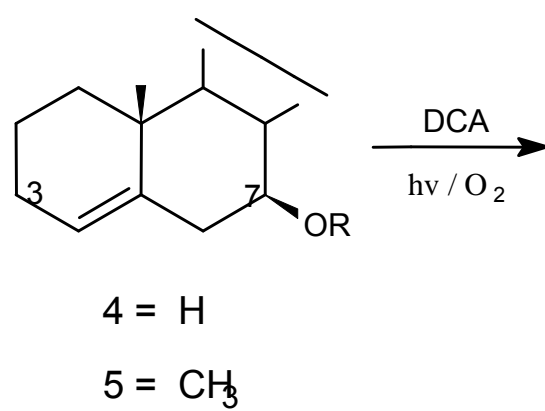

Scheme 3.<smiles>[R]C1C[C@]2(O)C=CCC[C@]2(C)C(C)C1C</smiles>

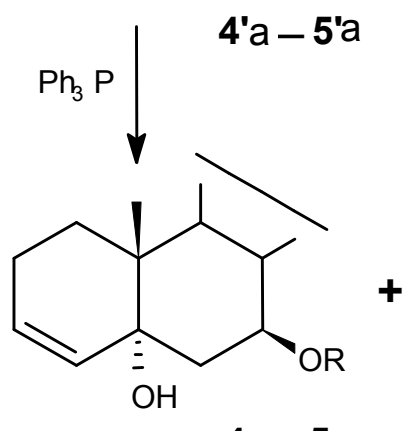

$4 a-5 a$<smiles>[R]O[C@H]1CC2=C[C@@H](O)CC[C@]2(C)[C@H](C)[C@@H]1C</smiles>

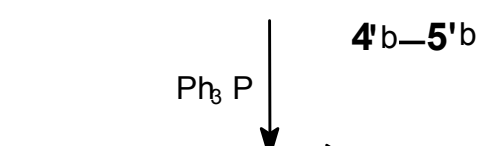<smiles>[R20]C1CC2=C[C@@H](O)CC[C@]2(C)[C@H](C)[C@H]1C</smiles>

$4 b-5 b$ 
This resemblance reflects that the ene reaction of ${ }^{1} \mathrm{O}_{2}$ with endocyclic $\mathrm{C}=\mathrm{C}$ migration in both cases (i.e. B ring in cholesterol and A ring in pseudocholesterol, respectively) occurs in the same ring to give $5 \alpha$ hydroperoxides and no exocyclic enol product was observed.

Since the photooxygenation of steroids is known to cause an increased brittleness of the cell membrane due to increased polarity of hydroxy and hydroperoxide products which leads to hemolysis [3], the preliminary results in this work for modulating photooxygenation of the title steroids via the ene reaction of ${ }^{1} \mathrm{O}_{2}$ may provide further understanding on their potential consequence of photochemical damage to $\Delta^{5}$-steroids.

\section{Experimental}

\section{Chemicals and instruments}

Cholestrol (1) was Fluka reagent grade, recrystallized from methanol, m.p.148-150 ${ }^{\circ} \mathrm{C} .3 \beta$-Methoxycholest-5-ene (2) was prepared as follows: (1) Cholesteryl tosylate: $38.6 \mathrm{~g}$ of $\mathbf{1}$ was dissolved in warm pyridine $(50 \mathrm{~mL})$, and then toluenesulfonyl chloride $(28.5 \mathrm{~g})$ was added. A white precipitate appeared after $5 \mathrm{~min}$. The mixture was left standing overnight and extracted with ethyl ether. Workup gave the product, m.p. 132-133 C (ref. [16] 131.5-132.5 C). The yield was $48 \mathrm{~g}$ (88\%). (2) Preparation of $3 \beta$-methoxy-cholest-5-ene 2: $2 \mathrm{~g}$ of Cholesteryl tosylate were dissolved in $50 \mathrm{~mL}$ of methanol and refluxed for $0.5 \mathrm{~h}$. A white precipitate appeared after cooling the solution. Workup gave 2, m.p. 81-82

C. The yield of 2 was $1.6 \mathrm{~g}\left(90 \%\right.$ ). $\mathrm{v}_{\max }$ : 2945, 2868, 1667 (w, C=C), 1455, 1188, 1103, $934 \mathrm{~cm}^{-1}$; m/z: $400\left(\mathrm{M}^{+}, 80 \%\right), 385\left(\mathrm{M}^{+}-\mathrm{Me}\right), 368\left(\mathrm{M}^{+}-\mathrm{MeOH}, 100 \%\right)$.

Cholesteryl acetate (3) was prepared according to the literature procedure [11]. Pseudocholesterol (4) was synthesized from cholesterol via a three-step reaction according to the literature method [12]. 7 $\beta$-Methoxypseudo-cholesterol-4-ene (5) was prepared by a similar procedure for 2. 9,10-Dicyanoanthracene (DCA) was Aldrich reagent grade, recrystallized from toluene, m.p. 324-326 C. Lumiflavin (LF) was prepared by a two-step reaction of riboflavin (RF) according to the literature method [13], m.p. 350-352 C (dec.); m/e: $256\left(\mathrm{M}^{+}\right), 242\left(\mathrm{M}^{+}-14,100 \%\right)$. Acetonitrile and pyridine were prepared by standard method and other reagents were used as purchased. ${ }^{1} \mathrm{H}-\mathrm{NMR}$ spectra were recorded on a Bruker AM 400 (TMS as internal standard). MS spectra were measured on a Finigan 4021. IR spectra were obtained on a Nicolet FT-IR 5DX. UV spectra were recorded on a Shimadzu UV-240. Melting points are uncorrected.

\section{Photooxygenations and product isolation}

Photolysis was carried out in a photochemical glass reactor equipped with a water-cooled jacket attached to an automatic controller. The light source was a $500 \mathrm{w}$ medium-pressure mercury lamp. The mixture of steroid, sensitizer and solvent in a glass tube was irradiated with continuous oxygen bubbling. The progress of reactions was monitored by TLC. 


\section{(1) LF-sensitized photooxygenation of $\mathbf{1}$ in $\mathrm{MeCN}$}

$77.2 \mathrm{mg}$ of 1 in MeCN solution $(80 \mathrm{~mL})$ containing $\mathrm{LF}\left(5 \times 10^{-4} \mathrm{~mol} \mathrm{dm}^{-3}\right)$ was irradiated for $4 \mathrm{~h}$ with oxygen bubbling, then $\mathrm{Ph}_{3} \mathrm{P}$ (100mg) was added and the mixture stirred for $0.5 \mathrm{~h}$. The solvent was evaporated and the mixture was chromatographed on silica gel eluting with toluene/ethyl acetate. The following three purified components were obtained: $15.2 \mathrm{mg}$ of recovered cholesterol (1); $12 \mathrm{mg}$ of cholest-6-en-3 $\beta$, 5 $\alpha$-diol (1a), recrystallized from methanol, m.p. 147-148 C (ref: 147-148 C [15]). $v_{\max }: 3600,3423(\mathrm{~b}, \mathrm{OH}), 1636(\mathrm{w}, \mathrm{C}=\mathrm{C}) \mathrm{cm}^{-1} ; \mathrm{m} / \mathrm{e}: 384\left(\mathrm{M}^{+}-\mathrm{H}_{2} \mathrm{O}, 30 \%\right), 366\left(\mathrm{M}^{+}-2 \mathrm{H}_{2} \mathrm{O}, 100 \%\right)$, 351, 143, $135(100 \%) ; \delta_{\mathrm{H}}\left(\mathrm{CDCl}_{3}\right): 0.71\left(\mathrm{~s}, 3 \mathrm{H}, 18-\mathrm{CH}_{3}\right), 0.93\left(\mathrm{~s}, 3 \mathrm{H}, 19-\mathrm{CH}_{3}\right), 4.13(\mathrm{~m}, 1 \mathrm{H}, 3-\mathrm{CH})$, $5.57(1 \mathrm{H}, \mathrm{dd}, \mathrm{J}=9.8,2.5 \mathrm{~Hz}, 6-\mathrm{H}), 5.63(1 \mathrm{H}, \mathrm{dd}, \mathrm{J}=9.8,1.7 \mathrm{~Hz}, 7-\mathrm{H}) ; 47 \mathrm{mg}$ of cholest-5-en-3 $\beta, 7(\alpha, \beta)-$ diol (1b), which was further separated by silica gel chromatograph to give cholest-5-en-3 $\beta, 7 \beta$-diol 1b$(\alpha)$, m.p. 176-178 $\quad$ C. $v_{\max }: 3368$ (b, OH), 2933, 2867 (s, C-H), 1662 (w, C=C), 1467, $1382 \mathrm{~cm}^{-1}$; m/e: $402\left(\mathrm{M}^{+}\right), 384\left(\mathrm{M}^{+}-\mathrm{H}_{2} \mathrm{O}\right), 369\left(\mathrm{M}^{+}-\mathrm{H}_{2} \mathrm{O}-\mathrm{CH}_{3}, 90 \%\right), 351\left(\mathrm{M}^{+}-2 \mathrm{H}_{2} \mathrm{O}-\mathrm{CH}_{3}\right), 247,135(100 \%) ; \delta_{\mathrm{H}}$ $\left(\mathrm{CDCl}_{3}\right): 0.69$ (s, 3H, 18- $\left.\mathrm{CH}_{3}\right), 1.02\left(\mathrm{~s}, 3 \mathrm{H}, 19-\mathrm{CH}_{3}\right), 3.60(\mathrm{~m}, 1 \mathrm{H}, 3-\mathrm{CH}), 3.87(\mathrm{~m}, 1 \mathrm{H}, 7-\mathrm{H}), 5.61$

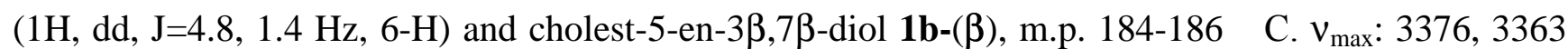
(b, OH), 2943, 2867 (s, C-H), 1662 (w, C=C), $1466 \mathrm{~cm}^{-1} ; \mathrm{m} / \mathrm{e}: 402\left(\mathrm{M}^{+}\right), 384\left(\mathrm{M}^{+}-\mathrm{H}_{2} \mathrm{O}, 100 \%\right), 369$ $\left(\mathrm{M}^{+}-\mathrm{H}_{2} \mathrm{O}-\mathrm{CH}_{3}, 40 \%\right), 351\left(\mathrm{M}^{+}-2 \mathrm{H}_{2} \mathrm{O}-\mathrm{CH}_{3}\right), 247,135 ; \delta_{\mathrm{H}}\left(\mathrm{CDCl}_{3}\right): 0.69\left(\mathrm{~s}, 3 \mathrm{H}, 18-\mathrm{CH}_{3}\right), 1.06(\mathrm{~s}, 3 \mathrm{H}$, 19- $\left.\mathrm{CH}_{3}\right), 3.58$ (m, 1H, 3-CH), 3.87 (m, 1H, 7-H), 5.30 (t, 1H, 6-H).

\section{(2) LF-sensitized photooxygenation of $\mathbf{1}$ in pyridine}

The same amount of $\mathbf{1}, \mathrm{LF}$ and solvent as in experiment (1) was used. Irradiation of the mixture for $6 \mathrm{~h}$ with oxygen bubbling was carried out, then a similar work-up and isolation as in (1) were employed. The following three purified compounds were obtained: $7.2 \mathrm{mg}$ of recovered 1, $59.2 \mathrm{mg}$ of $\mathbf{1 a}$ and $8.4 \mathrm{mg}$ of $\mathbf{1 b}$. Their spectra data were the same as those given in (1).

\section{(3) DCA -sensitized photooxygenation of 1 in $\mathrm{MeCN}$}

Compound 1 (77.2 mg; $0.03 \mathrm{mmol})$ in DCA-MeCN solution $\left(80 \mathrm{~mL}, 4 \times 10^{-4} \mathrm{~mol} \mathrm{dm}^{-3}\right)$ was irradiated for $6 \mathrm{~h}$ with oxygen bubbling, then a similar work-up and isolation procedure as in (1) were employed. The following three purified compounds were obtained: $8 \mathrm{mg}$ of recovered 1, $30.1 \mathrm{mg}$ of $\mathbf{1 a}$ and $39.8 \mathbf{m g}$ of $\mathbf{1 b}$ were obtained. Their spectra data were the same as those described in (1).

\section{(4) LF-sensitized photooxygenation of 2 in $\mathrm{MeCN}$}

Compound $2(80 \mathrm{mg})$ in an MeCN solution containing LF $\left(150 \mathrm{~mL} ; 5 \times 10^{-4} \mathrm{~mol} \mathrm{dm}^{-3}\right)$ was irradiated for $10 \mathrm{~h}$ with oxygen bubbling, then a similar work-up and isolation procedure as in (1) were used. The following three purified compounds were obtained: Recovered 3ß-methoxy-cholest-5-ene (2) (15.8

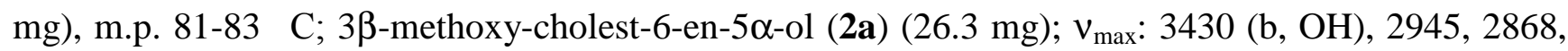
1665 (w, C=C), 1454, 1384, 1103, $1018 \mathrm{~cm}^{-1}$; m/e: $401\left(\mathrm{M}^{+}-\mathrm{Me}\right), 398\left(\mathrm{M}^{+}-\mathrm{H}_{2} \mathrm{O}, 100 \%\right), 384\left(\mathrm{M}^{+}-80\right.$ $\%), 353,301,210,71,43 ; \delta_{\mathrm{H}}\left(\mathrm{CDCl}_{3}\right): 0.70\left(\mathrm{~s}, 3 \mathrm{H}, 18-\mathrm{CH}_{3}\right), 1.19\left(\mathrm{~s}, 19-\mathrm{CH}_{3}\right), 3.36\left(\mathrm{~s}, 3 \mathrm{H}, \mathrm{OCH}_{3}\right)$, $3.70(\mathrm{~m}, 1 \mathrm{H}, 3-\mathrm{CH}), 5.65(\mathrm{~m}, 2 \mathrm{H}, 6-$ and $7-\mathrm{H})$ and $3 \beta$-methoxy-cholest-5-en-7 $(\alpha, \beta)$-ol (2b) (33.5 mg); $v_{\max }: 3430$ (b, OH), 2938, 2868, 1665 (w, C=C), 1468, 1384, $1101 \mathrm{~cm}^{-1} ; \mathrm{m} / \mathrm{e}: 416\left(\mathrm{M}^{+}\right), 401\left(\mathrm{M}^{+}-\mathrm{Me}\right)$, 
$398\left(\mathrm{M}^{+}-\mathrm{H}_{2} \mathrm{O}\right), 384\left(\mathrm{M}^{+}-\mathrm{MeOH}, 100 \%\right), 353,275,210,43 ; \delta_{\mathrm{H}}\left(\mathrm{CDCl}_{3}\right): 0.69\left(\mathrm{~s}, 3 \mathrm{H}, 18-\mathrm{CH}_{3}\right), 1.25((\mathrm{~s}$,

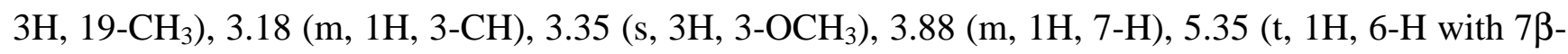
ol), $5.70(\mathrm{~d}, 1 \mathrm{H}, 6-\mathrm{H}$ with $7 \alpha-\mathrm{ol})$.

(5) DCA-sensitized photooxygenation of 2

Compound $2(80 \mathrm{mg})$ in MeCN solution containing DCA $\left(150 \mathrm{~mL} ; 4 \times 10^{-4} \mathrm{~mol} \mathrm{dm}^{-3}\right)$ was irradiated for $8 \mathrm{~h}$ with oxygen bubbling, then a similar work-up and isolation procedure as in (1) were employed. The following three purified compounds were obtained: 2 (12 $\mathrm{mg}$, recovered), $\mathbf{2 a}(28.2 \mathrm{mg})$ and $\mathbf{2 b}$ (39.2 mg).

(6) DCA-sensitized photooxygenation of $\mathbf{3}$

Compound $3(88.8 \mathrm{mg})$ in MeCN solution containing DCA $\left(100 \mathrm{~mL} ; 4 \times 10^{-4} \mathrm{~mol} \mathrm{dm}^{-3}\right)$ was irradiated for $7 \mathrm{~h}$ with oxygen bubbling, then a similar work-up and isolation procedure as in(1) were employed. The following three purified compounds were obtained: 3 (13.6 mg, recovered); $3 \beta$ acetyloxycholest-6-en-5 $\alpha$-ol (3a) (46.8 mg); $v_{\max }$ : 3507, 3451 (b, OH), 2945, 2868, 1728 (s, C=O), $1672(\mathrm{w}, \mathrm{C}=\mathrm{C}), 1458,1377,1243 \mathrm{~cm}^{-1}$; m/e: $443\left(\mathrm{M}^{+}-1\right), 426\left(\left(\mathrm{M}^{+}-\mathrm{H}_{2} \mathrm{O}\right), 411\left(\mathrm{M}^{+}-\mathrm{H}_{2} \mathrm{O}-\mathrm{CH}_{3}\right), 382\right.$, $367(100 \%), 351 ; \delta_{\mathrm{H}}\left(\mathrm{CDCl}_{3}\right): 0.68\left(\mathrm{~s}, 3 \mathrm{H}, 18-\mathrm{CH}_{3}\right), 1.26\left(\mathrm{~s}, 3 \mathrm{H}, 19-\mathrm{CH}_{3}\right), 2.04\left(\mathrm{~s}, 3 \mathrm{H}, 3-\mathrm{CH}_{3} \mathrm{COO}\right)$, $2.37(\mathrm{~m}, 1 \mathrm{H}), 4.98(\mathrm{~m}, 1 \mathrm{H}, 3-\mathrm{CH}), 5.62(\mathrm{~s}, 2 \mathrm{H}, 6$ - and 7-H); 3 $\beta$-acetyloxy-cholest-5-en-7( $\alpha, \beta)$-ol $(3 \mathbf{b})$ (22.3 mg); $v_{\max }: 3479$ (b, OH), 2945, 2868, 1735 (s, C=O), 1672 (w, C=C), 1451, 1370, $1032 \mathrm{~cm}^{-1}$; m/e: $443\left(\mathrm{M}^{+}-1\right), 426\left(\mathrm{M}^{+}-\mathrm{H}_{2} \mathrm{O}\right), 411,399,383,367,351,174,57,43 ; \delta_{\mathrm{H}}\left(\mathrm{CDCl}_{3}\right): 0.68(\mathrm{~s}, 3 \mathrm{H}, 18-$ $\left.\mathrm{CH}_{3}\right), 1.26\left(\mathrm{~s}, 3 \mathrm{H}, 19-\mathrm{CH}_{3}\right), 2.04\left(\mathrm{~s}, 3 \mathrm{H}, 3-\mathrm{CH}_{3} \mathrm{COO}\right), 2.54(\mathrm{~m}, 1 \mathrm{H}), 3.89(\mathrm{~m}, 1 \mathrm{H}, 7-\mathrm{CH}), 4.76(\mathrm{~s}, 1 \mathrm{H}$, $3-\mathrm{CH}), 5.35$ (s, 1H, 6-H with 7 $\beta$-ol), 5.72 (s, 1H, 6-H with $7 \alpha-\mathrm{ol})$.

\section{(7) LF-sensitized photooxygenation of $\mathbf{3}$ in $\mathrm{MeCN}$}

Compound $3(86.7 \mathrm{mg})$ in an MeCN solution containing $\mathrm{LF}\left(100 \mathrm{~mL} ; 5 \times 10^{-4} \mathrm{~mol} \mathrm{dm}^{-3}\right)$ was irradiated for $12 \mathrm{~h}$ with oxygen bubbling, then a work-up and isolation procedure similar to those described in (1) were employed. The following three purified compounds were obtained: recovered $\mathbf{3}$ (22.5 mg), 3a (42.2 mg) and $\mathbf{3 b}(18 \mathrm{mg})$.

\section{(8) DCA-sensitized photooxygenation of $\mathbf{4}$}

Compound $4(80 \mathrm{mg})$ in MeCN solution containing DCA $\left(100 \mathrm{~mL} ; 4 \times 10^{-4} \mathrm{~mol} \mathrm{dm}^{-3}\right)$ was irradiated for $5 \mathrm{~h}$ with oxygen bubbling, then a similar work-up and isolation procedure as in (1) were employed. The following three purified compounds were obtained: recovered compound 4 (42.3 mg); pseudocholest-3-en-5 $\alpha$-7 $\beta$-diol (4a) (21 mg); $v_{\text {max }}$ : 3382 (b, OH), 2931, 2840 (s, C-H), 1637 (w, C=C), $1454 \mathrm{~cm}^{-1}$; m/e: $384\left(\left(\mathrm{M}^{+}-\mathrm{H}_{2} \mathrm{O}, 24 \%\right), 369\left(\mathrm{M}^{+}-\mathrm{H}_{2} \mathrm{O}-\mathrm{CH}_{3}, 100 \%\right), 351\left(\mathrm{M}^{+}-2 \mathrm{H}_{2} \mathrm{O}-\mathrm{CH}_{3}\right), 233,135\right.$ $(100 \%) ; \delta_{\mathrm{H}}\left(\mathrm{CDCl}_{3}\right): 0.75\left(\mathrm{~s}, 3 \mathrm{H}, 18-\mathrm{CH}_{3}\right), 1.22\left(\mathrm{~s}, 3 \mathrm{H}, 19-\mathrm{CH}_{3}\right), 4.09(\mathrm{~m}, 1 \mathrm{H}, 7-\mathrm{CH}), 5.74(\mathrm{~m}, 2 \mathrm{H}, 3-$, 4-H); Pseudocholest-4-en-3 ( $\alpha, \beta)-7 \beta$-diol (4b) (5.2 mg); $v_{\max }$ : 3353 (b, OH), 2938, 2850 (s, C-H), 1645 (w, C=C), $1454 \mathrm{~cm}^{-1}$; m/e: $402\left(\mathrm{M}^{+}\right), 384\left(\mathrm{M}^{+}-\mathrm{H}_{2} \mathrm{O}, 100 \%\right), 369\left(\mathrm{M}^{+}-\mathrm{H}_{2} \mathrm{O}-\mathrm{CH}_{3}\right), 351\left(\mathrm{M}^{+}-2 \mathrm{H}_{2} \mathrm{O}-\right.$ 
$\left.\mathrm{CH}_{3}\right), 247,135 ; \delta_{\mathrm{H}}\left(\mathrm{CDCl}_{3}\right): 0.72\left(\mathrm{~s}, 3 \mathrm{H}, 18-\mathrm{CH}_{3}\right), 1.22\left(\mathrm{~s}, 3 \mathrm{H}, 19-\mathrm{CH}_{3}\right), 3.54(\mathrm{~m}, 1 \mathrm{H}, 7-\mathrm{CH}), 3.80(\mathrm{~m}$, $1 \mathrm{H}, 3-\mathrm{CH}), 5.35$ (s, $1 \mathrm{H}, 4-\mathrm{H}$ with $3 \beta-\mathrm{ol}), 5.78$ (d, 1H, 4-H with $3 \alpha-\mathrm{ol})$.

(9) DCA-sensitized photooxygenation of 5

Compound $5(80 \mathrm{mg})$ in MeCN solution containing DCA $\left(100 \mathrm{~mL} ; 4 \times 10^{-4} \mathrm{~mol} \mathrm{dm}^{-3}\right)$ was irradiated for $7 \mathrm{~h}$ with oxygen bubbling, then a similar work-up and isolation gave three compounds: recovered $\mathbf{5}$ (40.5 mg); 7 $\beta$-methoxy-pseudocholest-3-en-5 $\alpha$-diol (5a) $\left(21.7 \mathrm{mg}\right.$ ); $v_{\max }$ : 3410 (b, OH), 2942, 2870, $1662(\mathrm{w}, \mathrm{C}=\mathrm{C}), 1382 \mathrm{~cm}^{-1}$; m/e: $401\left(\mathrm{M}^{+}-\mathrm{Me}\right), 398\left(\mathrm{M}^{+}-\mathrm{H}_{2} \mathrm{O}, 100 \%\right), 384\left(\mathrm{M}^{+}-\mathrm{MeOH}\right), 366,351 ; \delta_{\mathrm{H}}$ $\left(\mathrm{CDCl}_{3}\right): 0.72\left(\mathrm{~s}, 3 \mathrm{H}, 18-\mathrm{CH}_{3}\right), 1.21\left(\mathrm{~s}, 3 \mathrm{H}, 19-\mathrm{CH}_{3}\right), 3.36\left(\mathrm{~s}, 3 \mathrm{H}, 7-\mathrm{OCH}_{3}\right), 3.74(\mathrm{~m}, 1 \mathrm{H}, 7-\mathrm{CH}), 5.64$ (m, 2H, 3-, 4-H); 7 $\beta$-methoxy-pseudocholest-4-en-3 ( $\alpha, \beta)$-ol (5b) (6.8 mg); $v_{\max }: 3410$ (b, OH), 2935, 2868 (s, C-H), 1670 (w, C=C), $1380 \mathrm{~cm}^{-1} ; \mathrm{m} / \mathrm{e}: 416\left(\mathrm{M}^{+}\right), 401\left(\mathrm{M}^{+}-\mathrm{Me}\right), 398\left(\left(\mathrm{M}^{+}-\mathrm{H}_{2} \mathrm{O}\right), 384\left(\mathrm{M}^{+}-\right.\right.$ $\mathrm{MeOH}, 100 \%), 366,351 ; \delta_{\mathrm{H}}\left(\mathrm{CDCl}_{3}\right): 0.73$ (s, 3H, 18- $\left.\mathrm{CH}_{3}\right), 1.21$ (s, 3H, 19- $\left.\mathrm{CH}_{3}\right), 3.20$ (m, 1H, 7$\mathrm{CH}), 3.37\left(\mathrm{~s}, 3 \mathrm{H}, 7-\mathrm{OCH}_{3}\right), 3.84(\mathrm{~m}, 1 \mathrm{H}, 7-\mathrm{CH}), 5.30$ (s, 1H, 4-H with 3ß-ol), 5.65 (d, 1H, 4-H with $3 \alpha-\mathrm{ol})$.

\section{Acknowledgements}

This work is supported by the National Natural Science Foundation of China (NNSFC, project No. 29772025).

\section{References}

1. Morrison, H. Ed. Biological Photochemistry, John Wiley \& Sons, Inc., New York, 1990.

2. Foote, C. S. in Free Radicals in Biology, ed by Pryor, W. A., Vol. 2, Academic Press, New York, 1976, pp.85.

3. Litnack, G., Biochemical Actions of Hormones, Vol. IX, Academic Press, New York, 1982.

4. Denny, R. W. Organic Reactions, Vol. 20, Dauban, W. G., ed., Wiley, New York, 1973, pp 133.

5. Jiang, Z. Q. Res. Chem. Intermed. 1990, 14, 185.

6. Mattes, S. L.; Farid, S. Org. Photochem. 1983, 6, 233.

7. Araki, Y.; Dobrowolski, D. C.; Goyne, T. E.; Hanson, D.C.; Jiang, Z. Q.; Lee, K. T.; Foote, C. S. J. Am. Chem. Soc. 1984, 106, 4570.

8. Jiang, Z. Q.; Zeng, D. X.; Liu, Y. C. Нuaxue Xuebao 1980, 47, 194.

9. Teng, J. I.; Kulig, M. J.; Smith, L. L; Kan, G.; Van Lie, J. E. J. Org. Chem. 1973, 38, 119.

10. Beckwith, A. L. J.; Davies, A. G.; Davison, G. E.; Maccoll, A.; Mruzek, M. H. J. Chem. Soc. Perkin Trans. 2 1989, 815.

11. Perish, E. J.; Schroepfer, G. J. Jr. J. Lipid Res. 1981, 22, 859.

12. Petersen, Q. R.; Chen, C. T. J. Am. Chem. Soc. 1955, 55, 2557.

13. Scott, K. N.; Marcei, T. H. Can J. Chem. 1979, 57, 27.

14. Perrin, D. D.; Armarego, W. L. F.; Perrin, D. R. Purification of Laboratory Chemicals, Pergamon Elmsford, New York, 1966. 
15. Smith, L. L.; Teng, J. I.; Kulig, M. L.; Hill, F. L. J. Org. Chem. 1973, 38, 1763.

16. Wallis, E.S.; Fernholz, E.; Gephart, F.T. J. Am Chem. Soc. 1937, 59, 139.

Samples Availability: Samples are available from the authors.

(C) 2001 by MDPI (http://www.mdpi.org). Reproduction is permitted for noncommercial purposes. 